Supplement of Biogeosciences, 17, 3135-3147, 2020

https://doi.org/10.5194/bg-17-3135-2020-supplement

(C) Author(s) 2020. This work is distributed under

the Creative Commons Attribution 4.0 License.

(c) (1)

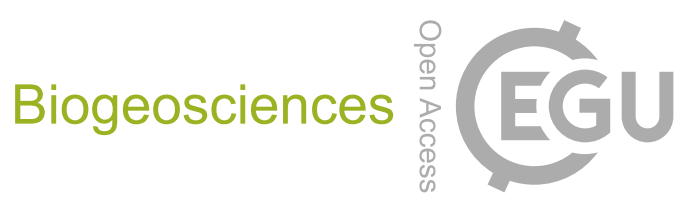

Supplement of

\title{
Vertical transport of sediment-associated metals and cyanobacteria by ebullition in a stratified lake
}

Kyle Delwiche et al.

Correspondence to: Sarah P. Preheim (sprehei1@jhu.edu) and Kyle Delwiche (kdelwiche@ seas.harvard.edu)

The copyright of individual parts of the supplement might differ from the CC BY 4.0 License. 


\section{S1 Supplemental Methods}

S1.1 Field experiments of cyanobacteria transport into bubble traps

We measured cyanobacteria transport in the field with bubble traps, but our measurements were contaminated by cyanobacteria in the surrounding water column. In Upper Mystic Lake, the cyanobacteria cell concentration varied with depth in the water column, with cell concentrations substantially lower at 10 and $11 \mathrm{~m}$ where the samples were collected than at the surface or near the bottom (Fig. S11). On average, the cyanobacteria cell concentration in the water surrounding the bubble trap was $8.3 \times 10^{3} \pm 5.2 \times 10^{3}$ cells $\mathrm{mL}^{-1}$. From direct cell measurements in the trap, we estimate about $1.5 \times 10^{4} \pm 6.3 \times 10^{3}$ cells $\mathrm{mL}^{-1}$ bubble volume. These estimates are more than an order of magnitude higher than estimates predicted by particle mass transport alone, which can be attributed to movement of cells from the surrounding water column into the trap as gas enters. We observed a strong negative correlation between the cell $\mathrm{mL}^{-1}$ bubble volume and the amount of gas in the trap (Fig. S12), which could be explained with a model of cell concentration in the trap from transport based on particles and water entering the trap from the surrounding water column at a rate of 9-12 $\mathrm{mL}$ of water per $\mathrm{mL}$ gas (see below).

\section{S1.2 Model of cell concentration in bubble trap}

Because of the bubble trap design, water from the surrounding water column enters as bubbles enter the trap contributing to the overall cyanobacteria cell number in the trap, through a process that can be modeled to fit the observations. The cell concentration in the trap is a combination of cells entering via bubblemediated transport and cells entering with input water from the surrounding water column. Although we tried to minimize the influence of the surrounding water column by placing the traps at a depth where cyanobacteria concentrations were lowest (e.g.10 and $11 \mathrm{~m}$; Fig. S11), there was still substantial contamination from the surrounding water column. To create a model explaining our field observations of a negative correlation between gas volume and cells $\mathrm{mL}^{-1}$ gas volume observed in the trap, we calculate the change in cells $\mathrm{mL}^{-1}$ in the trap with every $\mathrm{mL}$ gas entering the trap as:

$$
\frac{\Delta C_{k}}{\Delta V_{k}}=\frac{C_{g}+W \times \frac{C_{w c}}{F}-W \times \frac{C_{k-1}}{\left(500-V_{k-1}\right)}}{V_{t, k}}
$$

$\Delta C_{k}=$ Change in the number of cells in trap at $k \mathrm{~mL}$ of gas entering trap

$\Delta V_{k}=$ Change in the volume of gas in the trap at $k \mathrm{~mL}$ of gas entering trap (mL)

$V_{t, k}=$ Total volume of gas in the trap at $k \mathrm{~mL}$ of gas entering trap $(\mathrm{mL})$

$C_{g}=$ Cells transported into trap with bubbles at $k \mathrm{~mL}$ of gas entering trap (cells $\mathrm{mL}^{-1}$ gas)

$W=$ Volume of water entering and leaving the trap for each unit of gas entering (mL liquid $\mathrm{mL}^{-1}$ gas )

$C_{w c}=$ Cell concentration in surrounding water column (cells $\mathrm{mL}^{-1}$ liquid)

$F=$ Adjustment factor (unitless)

$C_{k-l}=$ Cells in trap at $k-1 \mathrm{~mL}$ of gas entering trap

$\mathrm{V}_{\mathrm{k}-1}=$ Volume of gas at unit $k-1 \mathrm{~mL}$ of gas entering trap $(\mathrm{mL})$

The cell number in the trap was calculated based on changes in cell number for each $k \mathrm{~mL}$ of gas entering trap from $1-120 \mathrm{~mL}$ in $1 \mathrm{~mL}$ increments based on Eq. S1. $F$ and $W$ for each $1 \mathrm{~mL}$ increment were manually adjusted to fit the observations. Only modeled volumes corresponding with field observations are plotted (Fig. S12), with model parameters as follows:

$C_{g}=880$ cells $\mathrm{mL}^{-1}$ gas

$W=12 \mathrm{~mL}$ liquid $\mathrm{mL}^{-1}$ gas

$C_{w c}=8,330$ cell $\mathrm{mL}^{-1}$ liquid

$F=3$

\section{S1.3 Caveats to quantitative polymerase chain reaction ( $q P C R$ ) and microscopy cell estimates}

Two methods of cell counting, quantitative polymerase chain reaction (qPCR) and microscopy, were used in this study, although we report only qPCR measurements of experimental samples because all of the experimental samples had cell concentrations well below the recommended 25-30 cells per field of view for microscopy measurements (Fry, 1990). Microscopy was used to determine the cell count for positive control cultures used to transform 16S rRNA copy number into cell counts (Fig. S4) and confirm the presence of cyanobacteria cells in all samples measured with qPCR (data not shown). Microscopy 
detects cyanobacteria cells based on auto-fluorescent pigments, although non-cyanobacterial cells could have a similar fluorescence spectrum, resulting in an over estimate of cyanobacteria with microscopy. Cyanobacteria in the lake could have genetic mismatches in the cyanobacteria-specific primer binding sites, resulting fewer cells estimated by qPCR. Cell counts from qPCR estimates of real samples could also be inaccurate (either high or low) if cyanobacteria in the lake do not have, on average, the same number of 16S rRNA gene copies per cell as our Microcystis stock.

\section{Supplemental References}

Fry, J. C.: Direct Methods and Biomass Estimation, Method Microbiol, 22, 41-85, 1990. 


\section{Supplemental Figures}
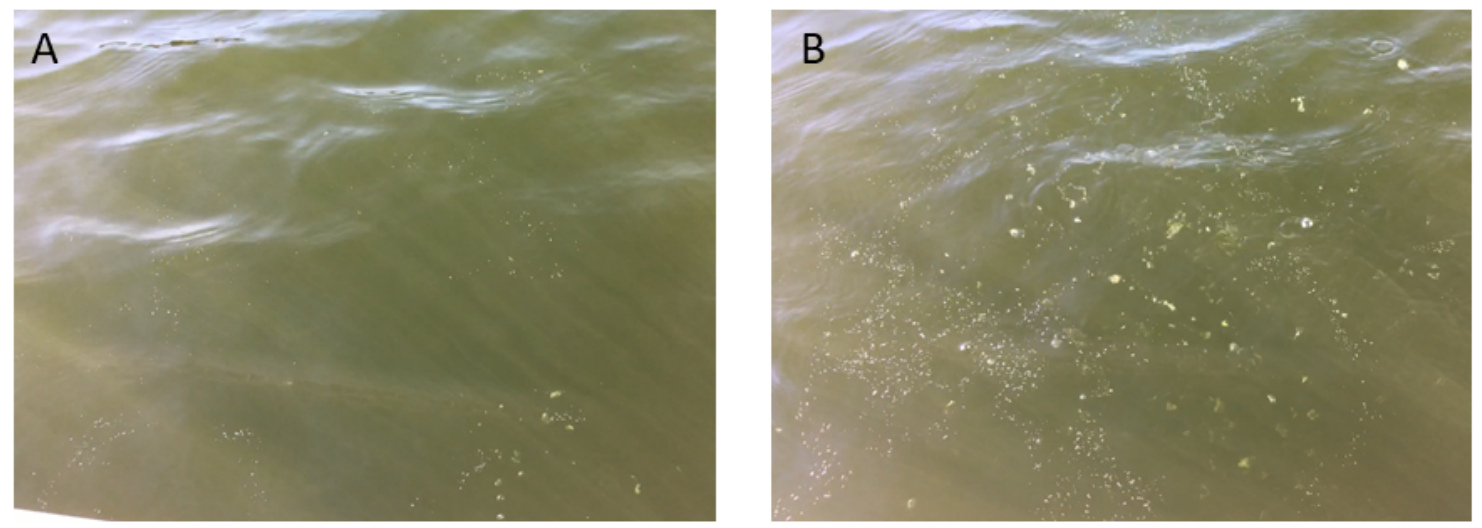

Figure S1. Picture of the lake surface near the beginning (A) and end (B) of a triggered bubble event at 15 $m$ depth showing an accumulation of particulate matter (visible as light specks on the water surface).

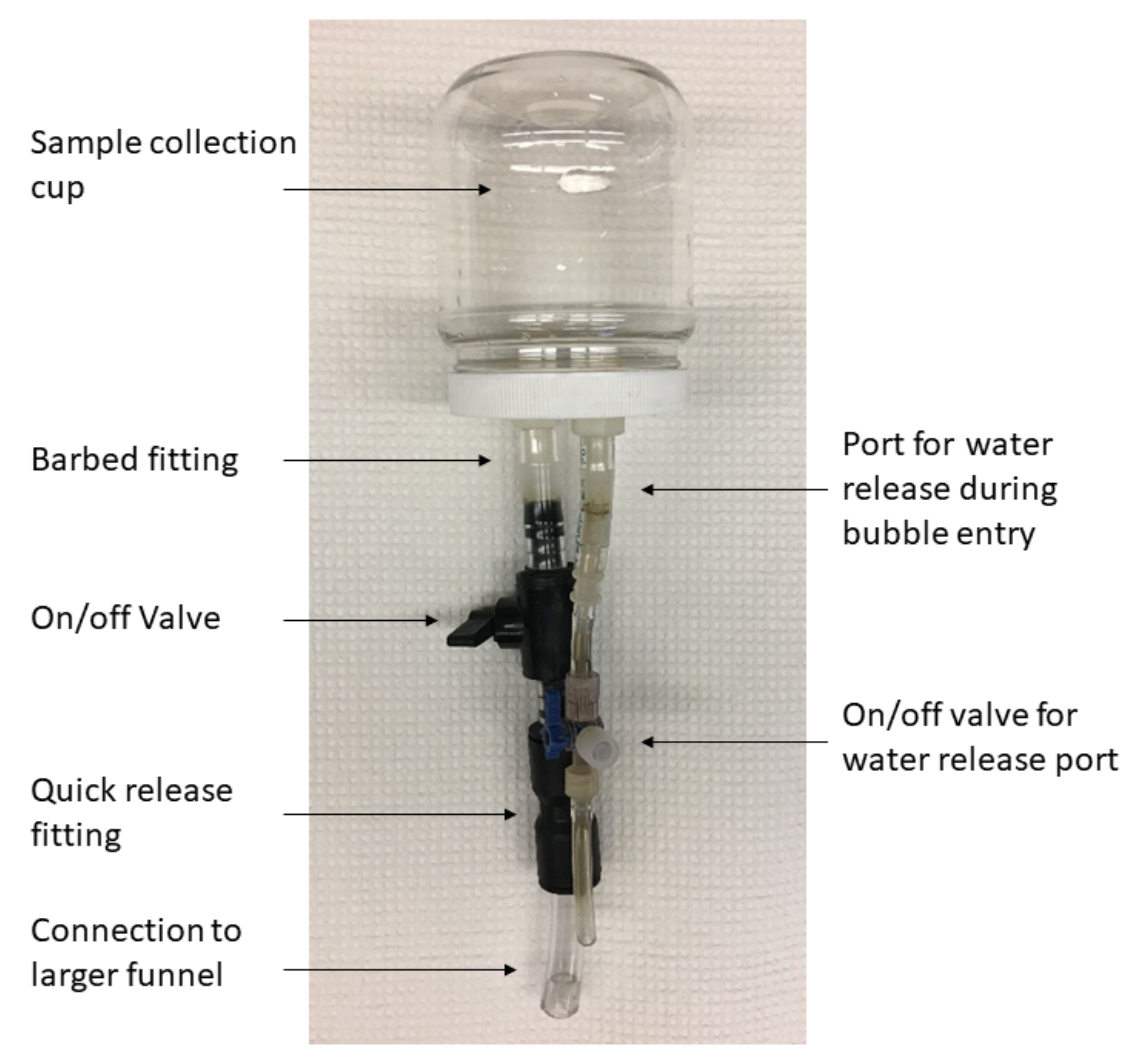

Figure S2. Particle sampling cup with on/off valves, a quick release fitting, and a secondary port that allows water to exit the cup when bubbles enter. 


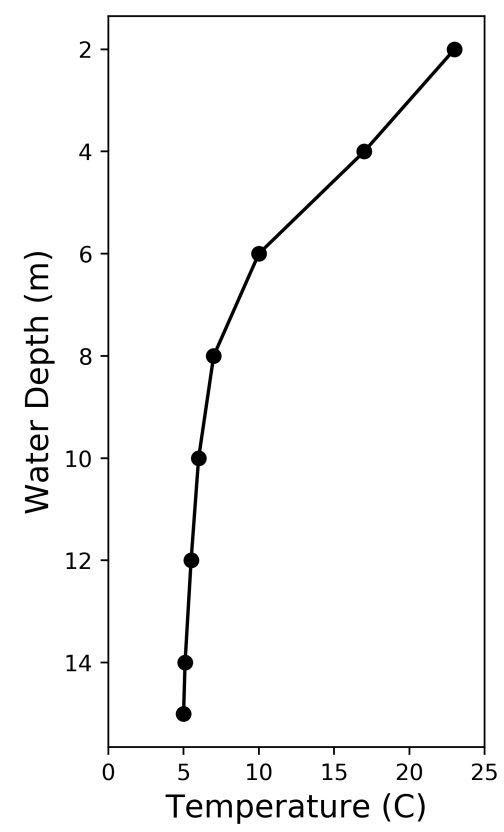

Figure S3. Water temperature profile taken during June 16, 2018 sampling event on Upper Mystic Lake.

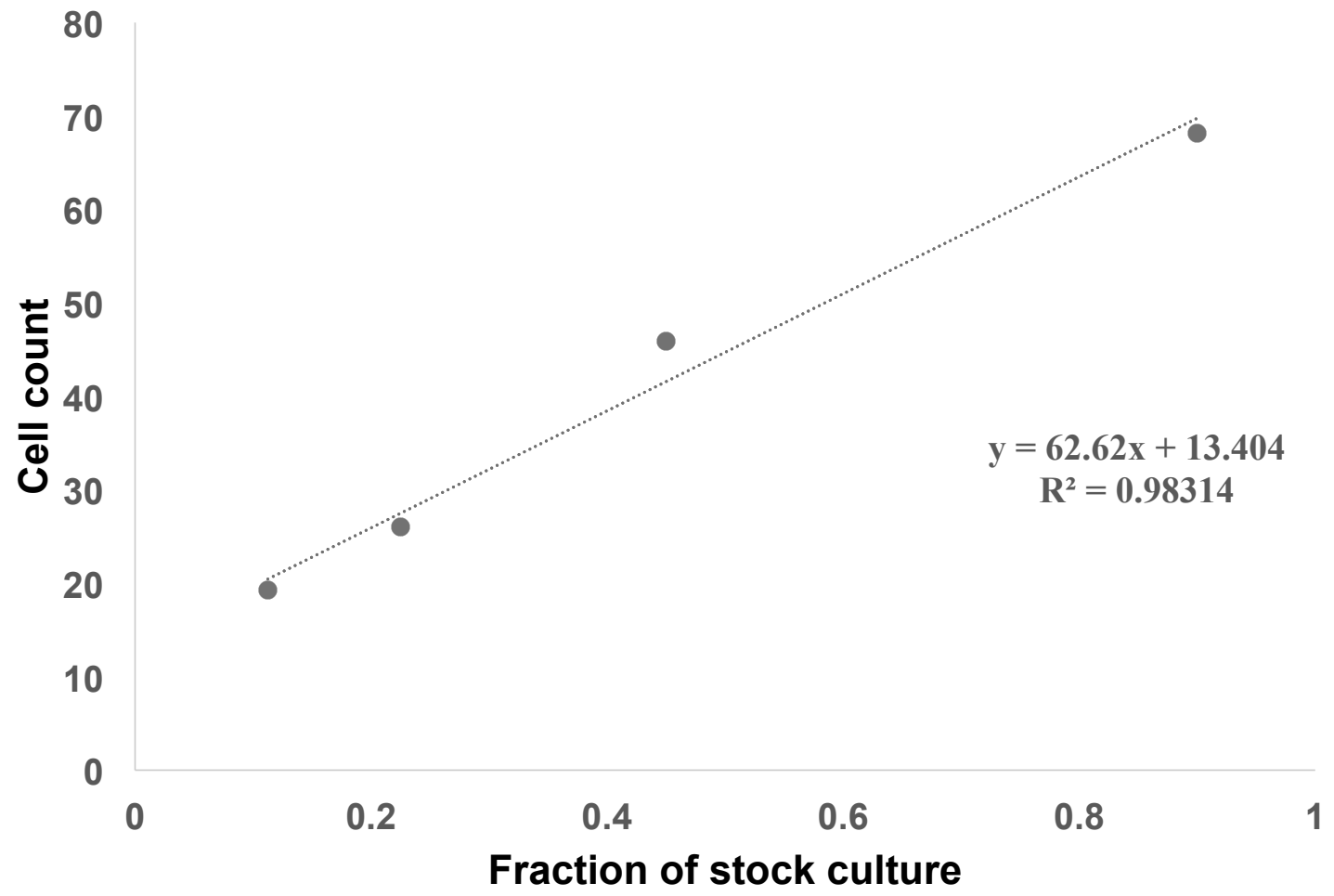


Figure S4. Dilution series of cultured Microcystis cells quantified by microscopy. Cells of Microcystis were diluted 2 -fold in $10 \%$ formalin and quantified using microscopy. 


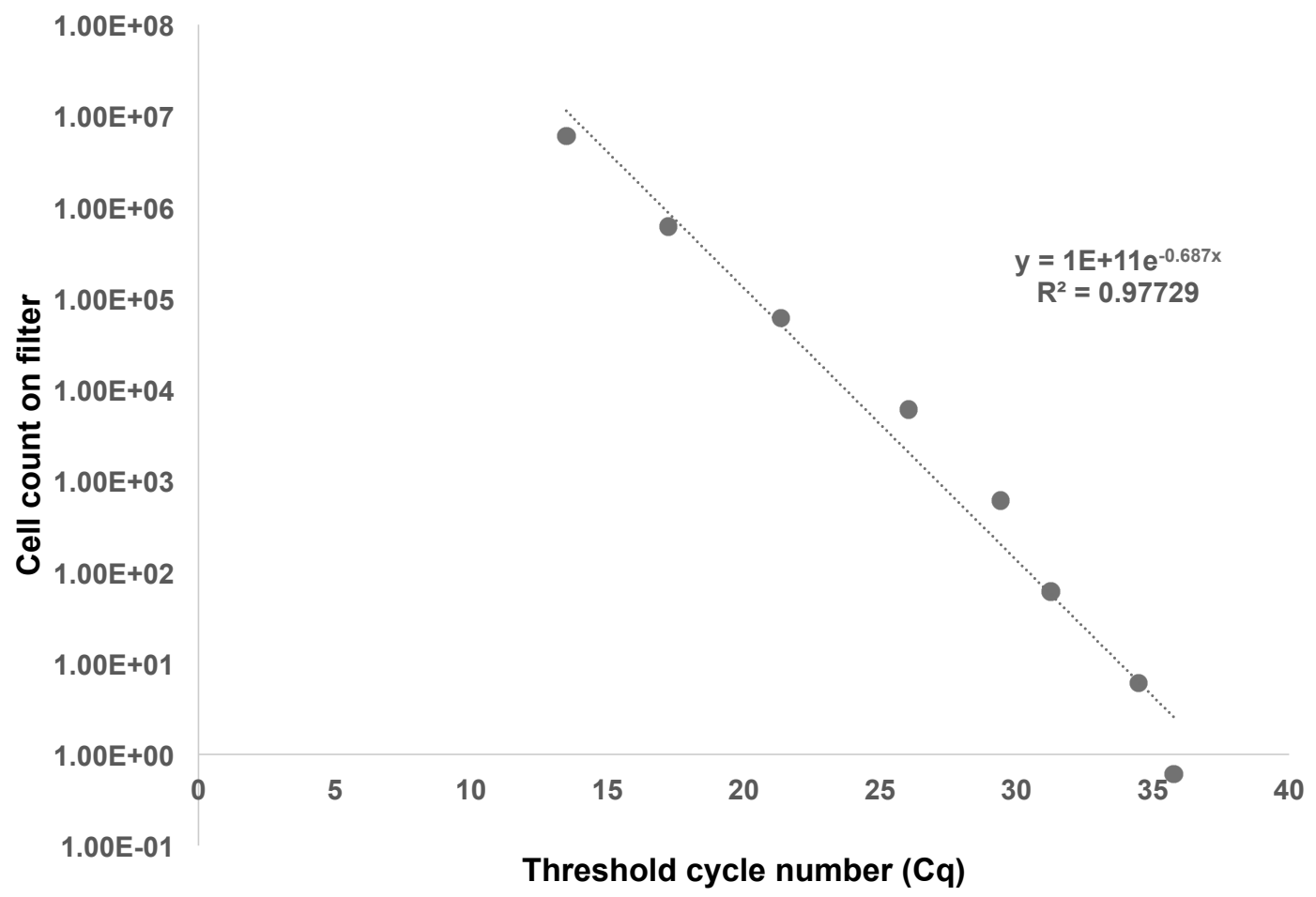

Figure S5: Serial dilution of Microcystis cells versus threshold values estimated by quantitative polymerase chain reaction (qPCR). Number of cells on the filter was estimated from the concentration of cells in the stock culture using microscopy times the dilution factor. This relationship was used to transform Cq values of experimental samples into Microcystis cell equivalents. 


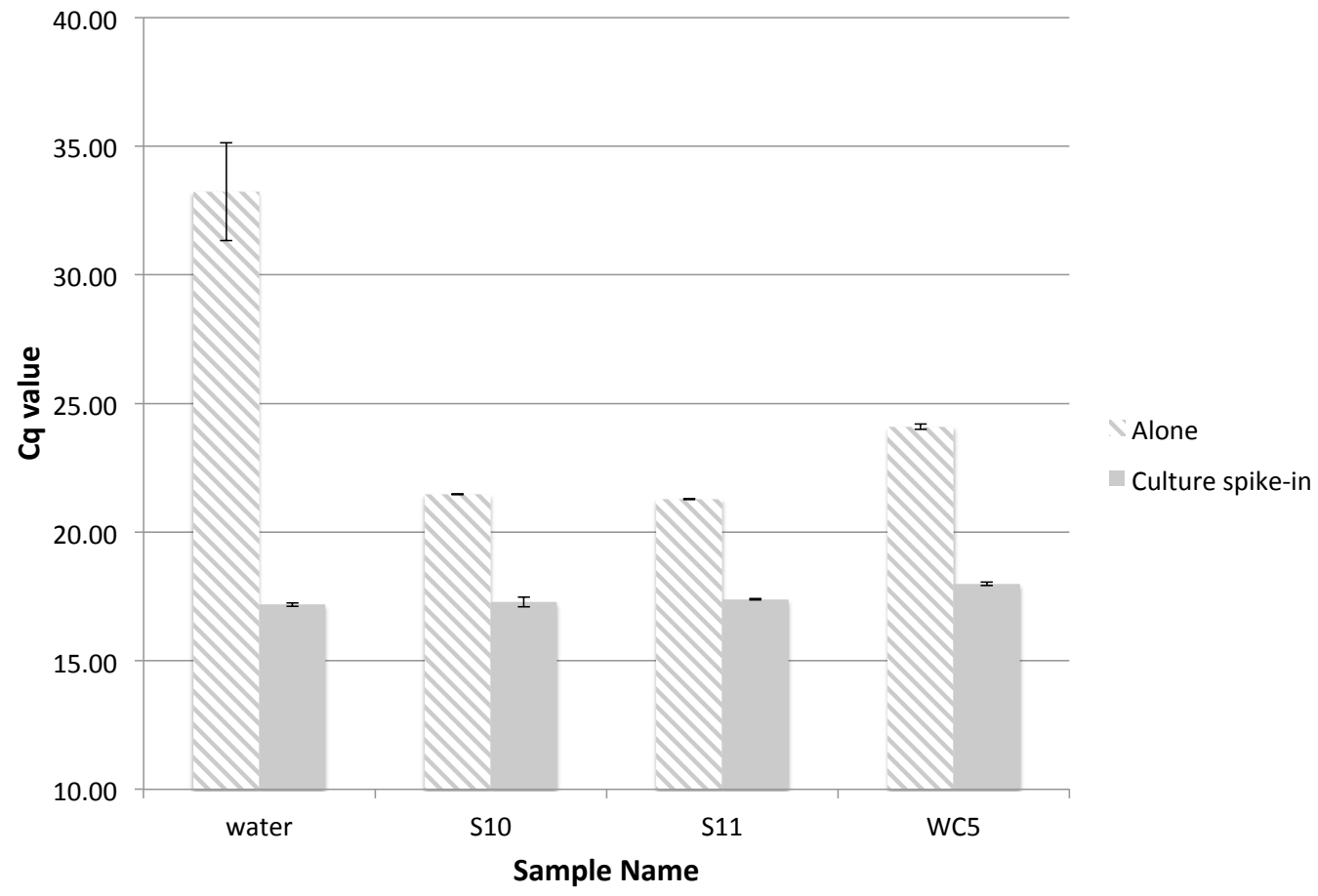

Figure S6. Inhibition in select samples was determined by adding Microcystis control culture DNA (spikein) to the qPCR reaction. Blue bars represent the threshold cycle number $(\mathrm{Cq})$ for the sample alone, while the red bars represent the threshold cycle number value after adding the spike-in control. Samples tested include a control (sterile water), bubble-trap samples (S10 and S11) and a water column sample (WC5). If contaminants in the extracted DNA are suppressing PCR, Cq values for spike-in samples (i.e. addition of Microcystis culture control) will be significantly higher than Cq value for the control (i.e. spike-in water sample). Instead, all spike-in samples had similar Cq values. 


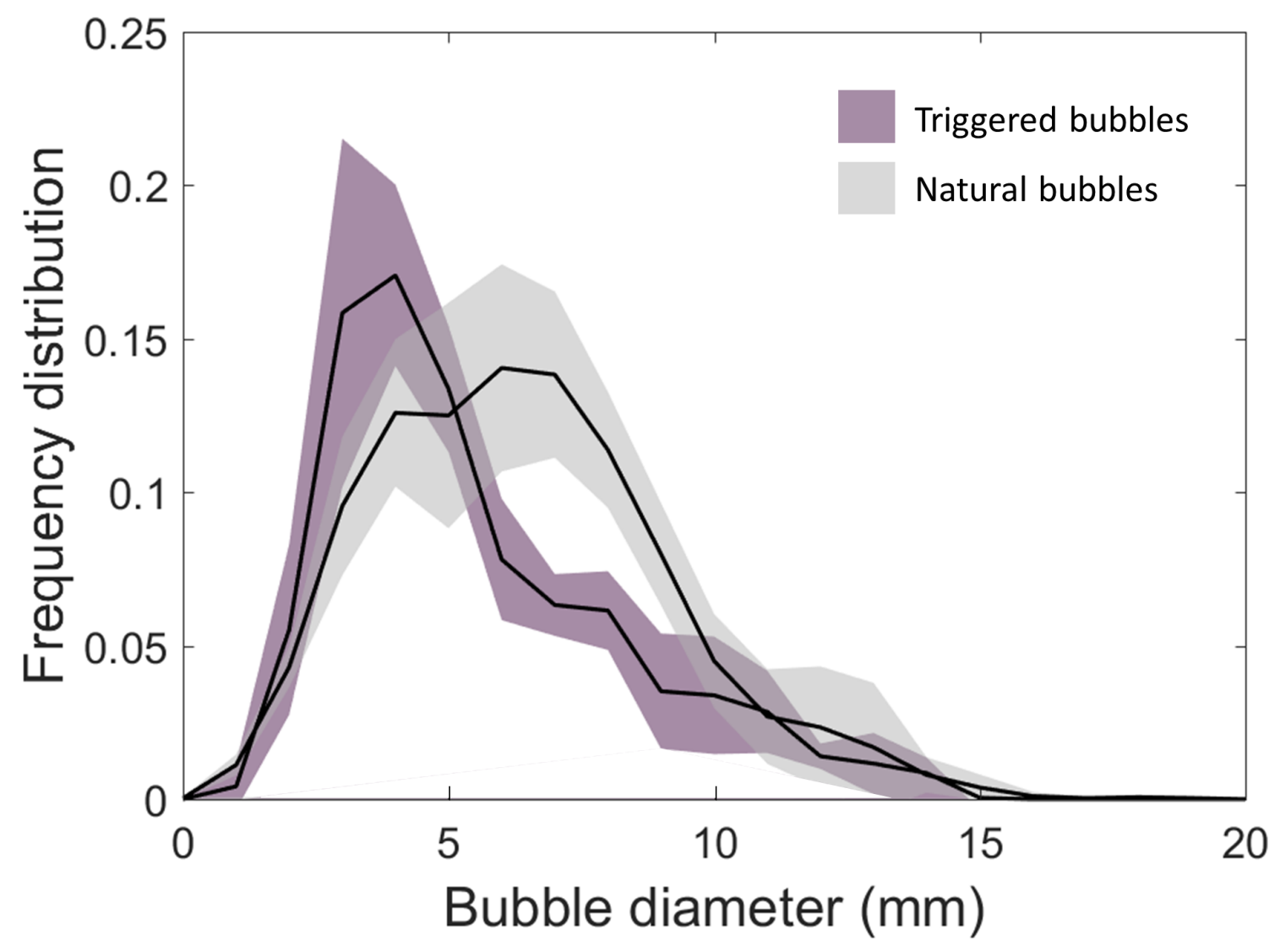

Figure S7. Frequency of occurrence of bubble diameter during triggered (purple) and natural (gray) events in Upper Mystic Lake. Mean (black lines) and standard deviation (shaded regions) for each event type. Bubbles were triggered by dropping an anchor multiple times during the October 2017 sampling event, while natural bubble size distribution are based on continuous measurements from the summer of 2015 and 2016 (Delwiche and Hemond, 2017). Frequency distribution numbers for the triggered bubbles are approximate because the bubble size sensor is unable to measure the rapid bubble flux that sometimes occurred with anchor-triggered bubble events. 

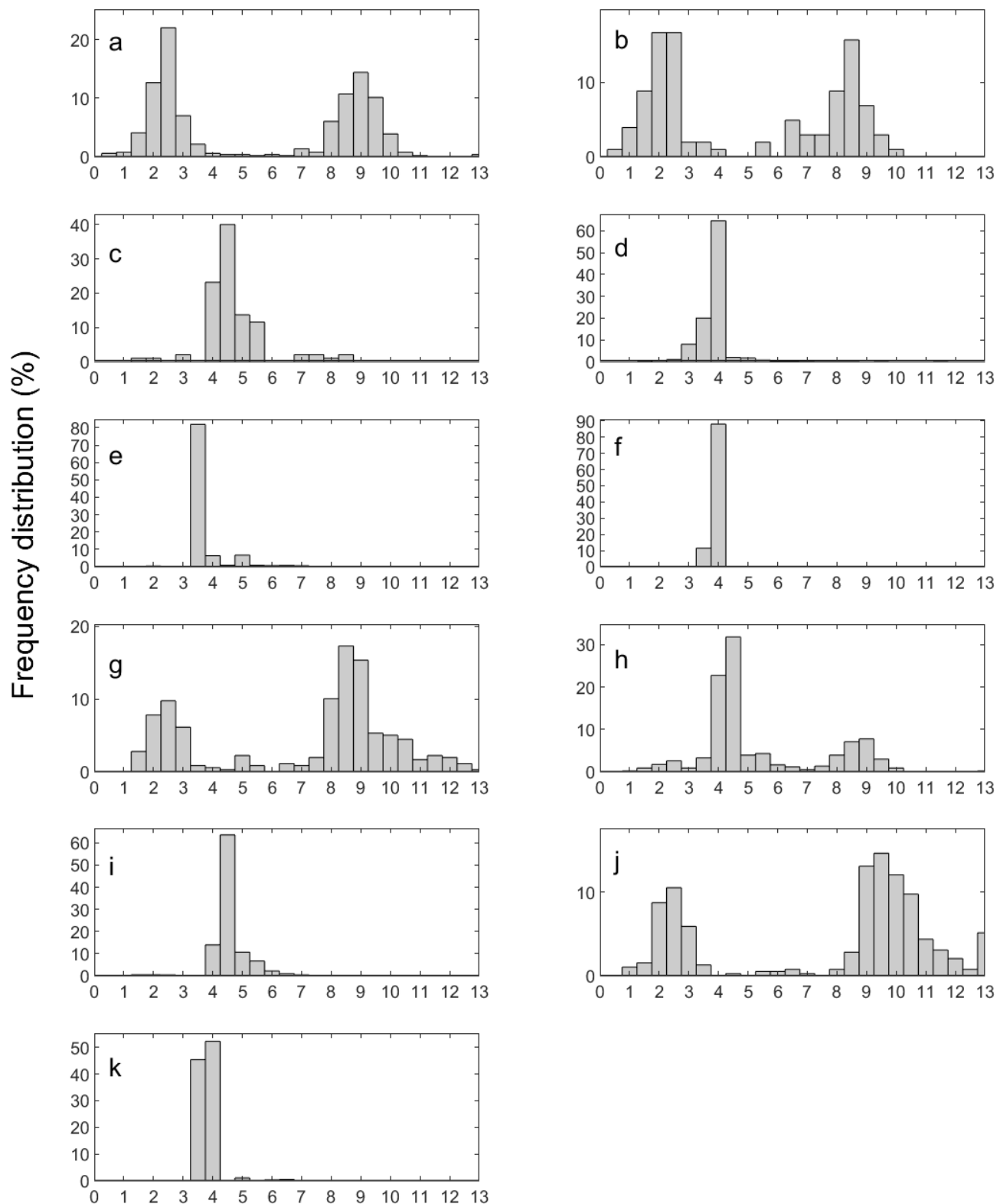

Bubble diameter $(\mathrm{mm})$

Figure S8. Frequency of bubble diameter observed across multiple trials (a-k) during the cyanobacteria experiment in the laboratory bubble column. Some trials had a bimodal diameter distribution. Panels $\mathrm{f}$ and $\mathrm{k}$ represent trials where air was bubbled directly above the sediment, and remaining panels represent trials where air was bubbled into the sediment. Note the different $y$-axis scales. 


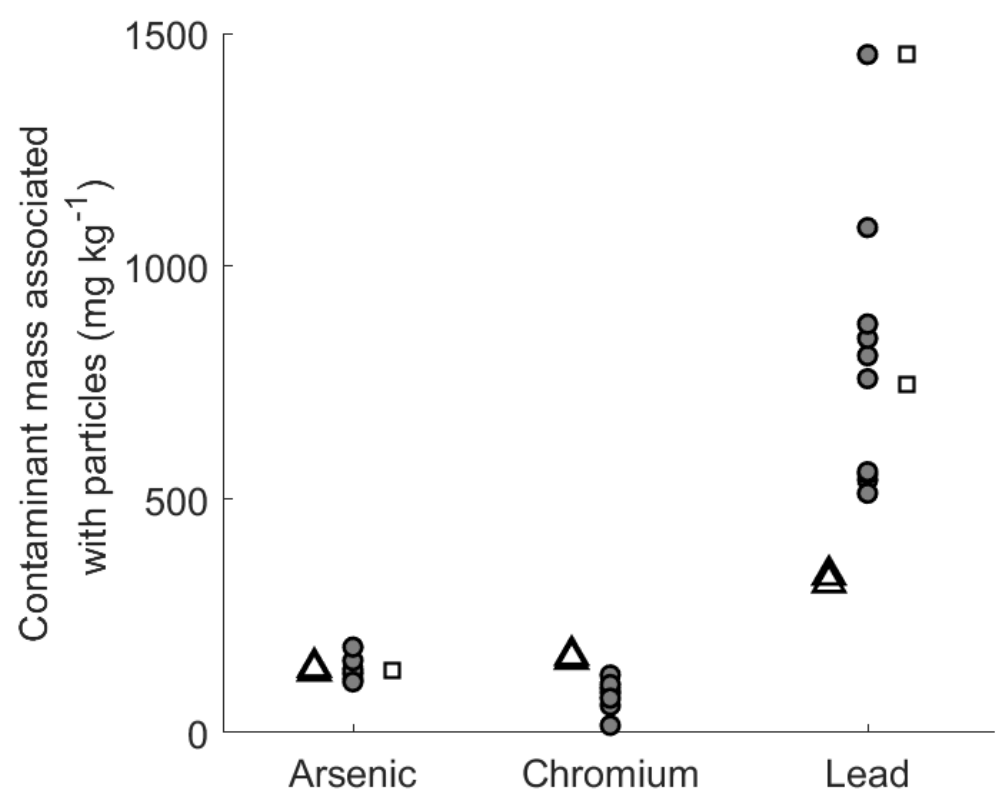

Figure S9. Comparison between mass of arsenic, chromium, and lead per $\mathrm{kg}$ of particulate matter for bubble column experiments and bulk sediment. Open triangles are ratios measured in sediment sample. Open squares are from samples where gas was emitted directly above the sediment and particulate matter was therefore scavenged from the water column. Error bars representing standard deviations are of same approximate size as symbols, and are therefore omitted for clarity. 

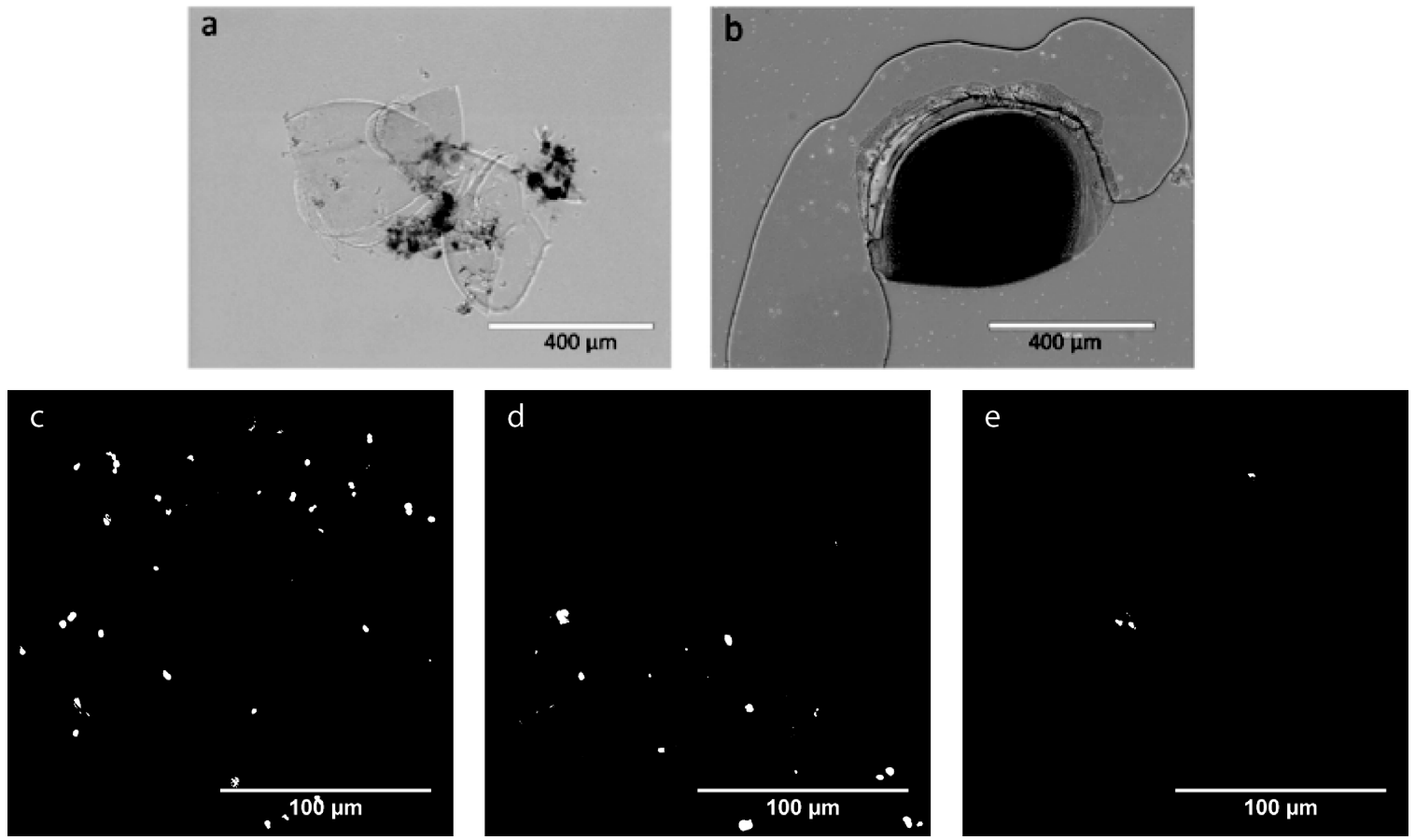

Figure S10. Microscope images of bubble-transported particles and cyanobacteria used as a control and

found in sediments and the bubble trap. Many microscopy images of particles found in the trap suggested they are of apparent biological origin, similar to Bosmina spp. remains (a) and Daphnia ephippia (b). Fluorescent microscopy of a cyanobacteria culture (Microcystis) was used to enumerate the total cyanobacteria cells as a positive control (c) and to determine the total number of cyanobacteria cells in sediments (d) and in the bubble trap (e). Microscopy images of cyanobacteria of cells found in the trap yielded few cells per field of view, which decreasing the accuracy of these measurements. Cyanobacteria were visualized by targeting the fluorescent properties of their photosynthetic pigments and translated into gray-scale images. The scale bar is found at the bottom right hand corner of each image. 


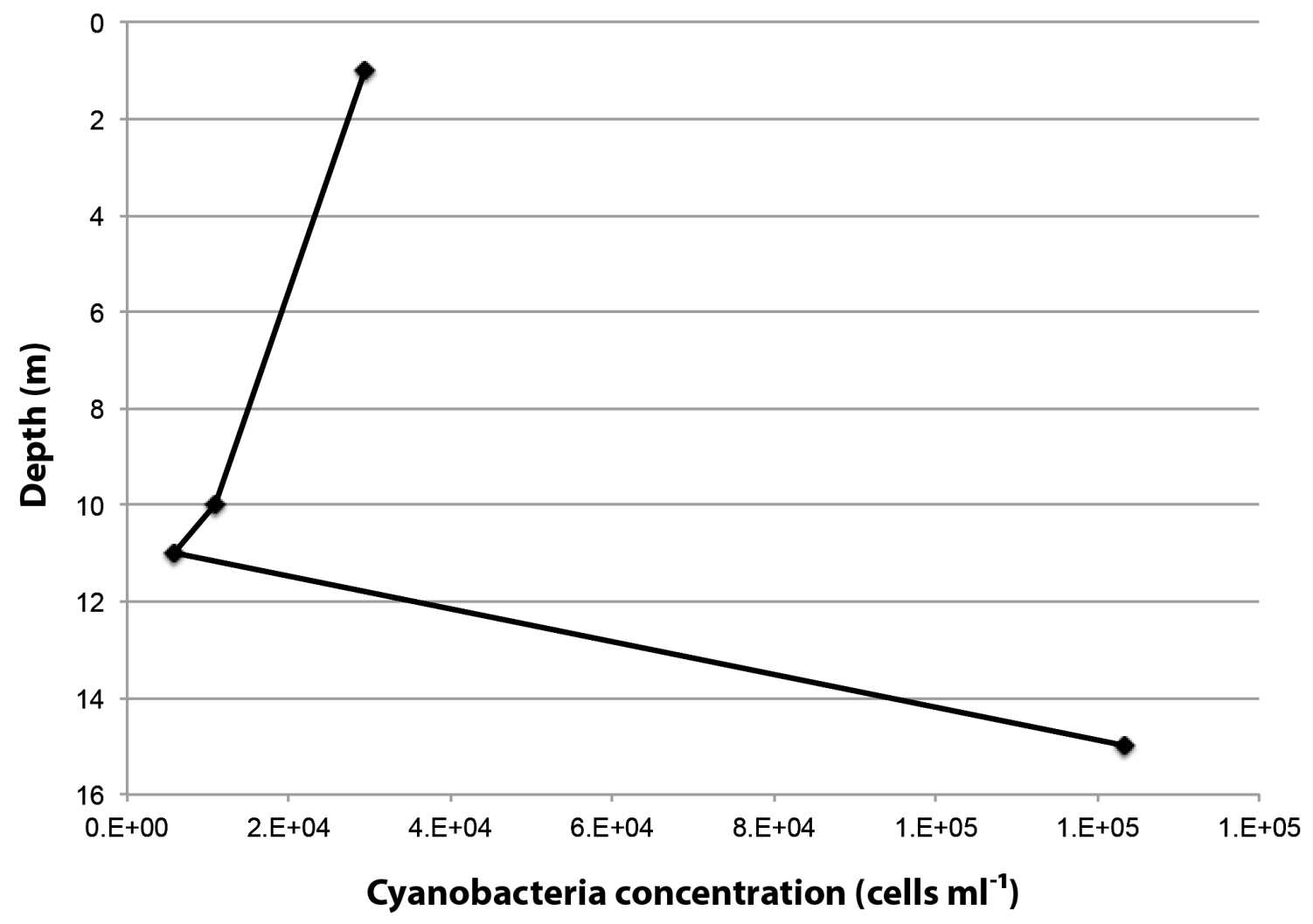

Figure S11. Concentration of cyanobacteria cells in water column at different depths as estimated with quantitative polymerase chain reaction. Cyanobacteria cell concentrations are lowest where the field bubble traps were placed, between 10-11 m. Even with water column cyanobacteria concentrations around the trap at their lowest concentrations observed in the lake, the contribution of cyanobacteria from the surrounding water column was sufficient to influence the bubble trap results. 


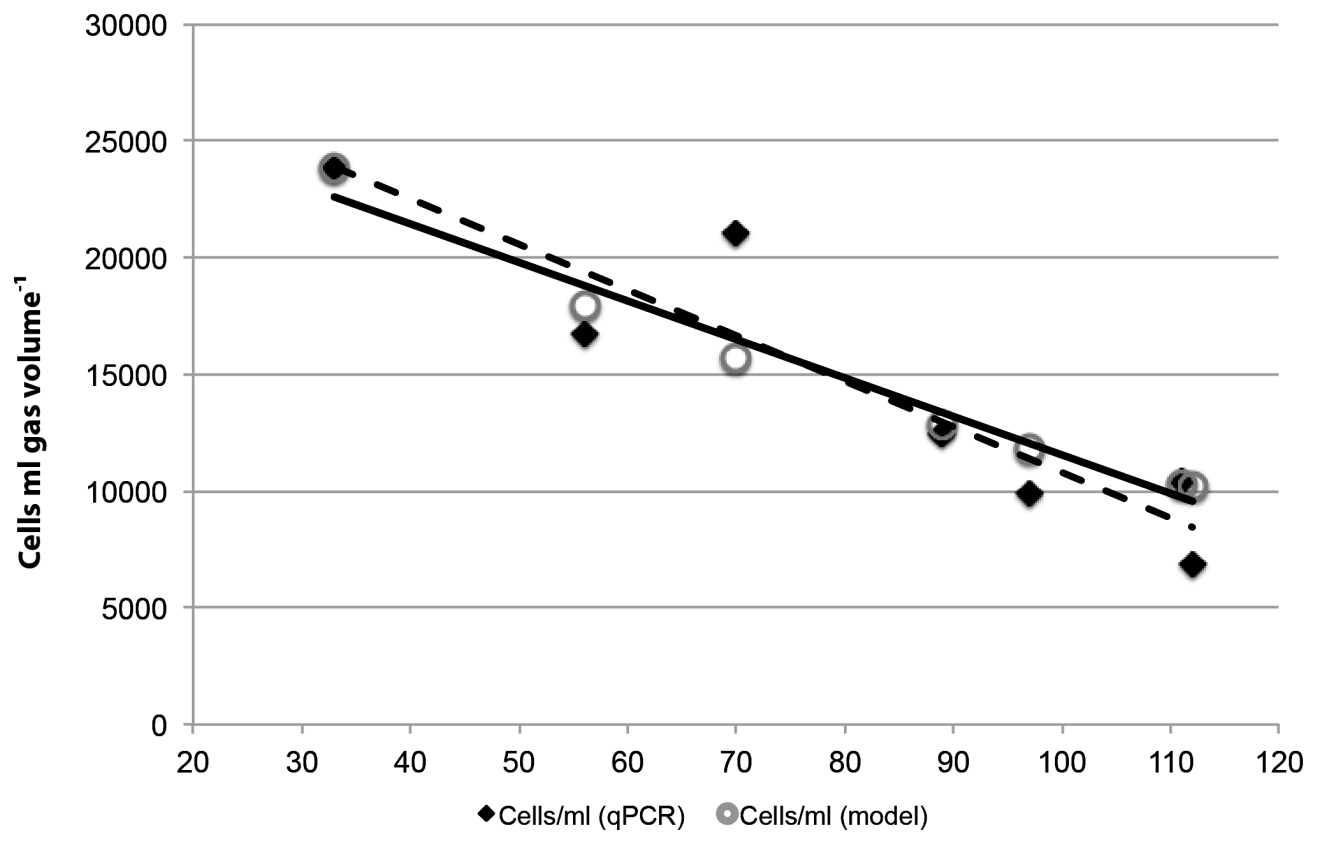

Gas volume (ml)

Figure S12. The negative correlation between the cyanobacteria cells $/ \mathrm{ml}$ bubble volume in the trap and the volume of gas in the trap observed from field measurements can be explained by a model of cells entering the trap from both the surrounding water column and with sediment transport from bubbling. Cells $/ \mathrm{ml}$ bubble volume in the trap based on qPCR measurements (black diamonds) is negatively correlated with total gas volume (dashed line; $\left.y=-195.42 x+30337 ; \mathrm{R}^{2}=0.85207\right)$. The model (gray circles), described in section $\mathrm{S} 1.2$, explains this relationship well (solid line; $\mathrm{y}=-164.94 \mathrm{x}+28037 ; \mathrm{R}^{2}=0.97302$ ). 